\title{
Modeling User Experience in Electronic Entertainment using Psychophysiological Measurements
}

\author{
Martin Čertický \\ Technical University in Košice \\ Email: martin.certicky@tuke.sk \\ (corresponding author)
}

Filippo Cavallo

The BioRobotics Institute, Scuola Superiore Sant'Anna

Email: filippo.cavallo@santannapisa.it

\author{
Michal Čertický \\ Czech Technical University in Prague \\ Email: certicky@agents.fel.cvut.cz
}

\author{
Peter Sinčák \\ Technical University in Košice \\ Email: peter.sincak@tuke.sk
}

\begin{abstract}
Analyses of user experience in electronic entertainment industry currently rely on self-reporting methods, such as surveys, ratings, focus group interviews, etc. We argue that selfreporting alone carries inherent problems - mainly the subject bias and interpretation difficulties - and therefore should not be used as a sole metric. To deal with this problem, we propose a possibility of creating a model of consumer experience based on psychophysiological measurements and describe how such model can be trained using machine learning methods. Models trained exclusively on real-time data produced by autonomic nervous system and involuntary physiological responses is not susceptible to subjective bias, misinterpretation and imprecision caused by the delay between the experience and the interview. This paper proposes a potentially promising direction for future research and presents an introductory analysis of available biological data sources, their relevance to user experience modeling and technical prerequisites for their collection. Multiple psychophysiological measurements (such as heart rate, electrodermal activity or respiratory activity) should be used in combination with selfreporting methods to prepare training sets for machine learning models. During our initial experiments, we collected time-series heart rate data for two computer games - Hearthstone and Dota 2. This preliminary analysis suggests the existence of a correlation between psychophysiological measurements and in-game events. Actual ready-to-use user experience models are out of the scope of this paper.
\end{abstract}

Keywords-Psychophysiological measurements, Modeling, Machine learning, Electronic entertainment, Correlation, Timeseries, Hearthstone, Dota 2

\section{ACKNOWLEDGMENT}

This work was supported by Slovak Research and Development Agency under the contracts No. APVV-16-0213 and No. APVV-015-0731

\section{INTRODUCTION}

Electronic entertainment (EE), especially video-games, has undergone an extensive growth over the past decade. In the USA alone (a country with the biggest video-game market in the world) the sales rose from $\$ 7.3$ billion in 2006 to $\$ 23.5$ billion spent in the game industry in 2015 . The global value of video-game industry is estimated to reach $\$ 120$ billion by 2019 [1]. Yet the assessment of user experience in video-games is still done using old-fashioned self-report techniques, such as questionnaires and interviews.

We propose an approach to assessing player experience more effectively while eliminating some disadvantages of selfreport techniques, such as untruthfulness or emotional bias.

Based on the assumption that psychophysiological measurements, such as heart rate, electrodermal activity or respiratory activity, correlate with user experience, we suggest combining them with the player's self-reports to create machine learningbased model of the player's experience. Such model may help developers predict how players will react to particular in-game events in the future.

\section{STATE OF THE ART}

Kivikangas et al. reviewed many scientific research papers concerning the use of psychophysiological measures in video-games [2]. The review investigated various types of psychophysiological metrics, pros and cons of research and particular causes of changes in subject's psychophysiological states. Another study relevant to our research is the work of Drachen et al. who studied a few correlations between selfreporting and psychophysiological measurements [3]. In their research, they used iGEQ (In-Game Experience Questionnaire) as a self-reporting method in combination with heart rate and electrodermal activity measures. The In-Game Experience Questionnaire (iGEQ) is a self-report scale for the exploration of the participant's experience during playing a computer game [4]. They found statistically significant correlations between heart rate (HR) and electrodermal activity (EDA) and iGEQ dimensions, although with different patterns of covariance. To prevent such covariance (considering more dimensions with a plan to use more psychophysiological measures) we plan to design our own questionnaire for future experiments. 
Drachen focused on the effects of particular game genres on the subject's physiological states [5]. The importance of game genre within our research is discussed in III. Further research of psychophysiological responses in video-games has focused on studying various effects of game genre and specific situations on the subject's psychophysiological states. For instance, Ballard and Wiest studied in 1996 the effects of violent video-games on the hostility and cardiovascular responses of males [6].

Codispoty et al. investigated various reactions to emotional movies with an emphasis on the subject's gender [7]. With a subject sample of 33 females and 27 males, they investigated cardiac response to highly arousing pleasant and unpleasant films. Based on the correlations found, they concluded that both pleasant and unpleasant arousing films prompt similar cardiac deceleration. Another aim of their study was to investigate differences in responses with regards to the subject's gender. However, their study only found differences between genders in subjective reports on films.

Future experiments will require an extensive number of subjects, since there are different results throughout individual types of players. Kallio et. al. established up to 9 different types of gaming mentalities [8]. To divide players into categories, they used heuristics such as Intensity (gameplay sessions length, regularity and concentration), Sociability (physical space, virtual space, outside game space) or Games (various devices, genres, etc.). Even though our subject sample most likely will not cover all of these categories, this is certainly a factor for future investigation.

One of our assumptions is that psychophysiological changes correlate with game events and the subject's emotions. Some work addressing this topic has already been published: Mandryk et al. used multiple psychophysiological measures in their experiments with players set in two different conditions, playing both against the computer and against another colocated player:

- EKG (Cardiovascular measures)

- HR (Heart Rate)

- GSR (Galvanic Skin Response)

- EMG (Electromyography)

- Resp Rate (Respiratory Rate)

- RespAmp (Respiratory Amplitude)

Afterwards, they were able to compile a list of possible correlations between particular psychophysiological measures and subjective responses [9]:

- Fun significantly correlated with GSR.

- Boredom correlated with EMG.

- Challenge correlated linearly with RespAmp and EMG.

- Ease also correlated linearly with RespAmp and EMG.

- Frustration significantly correlated with GSR and RespRate.

Several other papers have addressed the correlation problem of psychophysiological measures with similar results. Drachen, Nacke, Yannanakis and Petersen have produced several papers in the field, indicating how various emotions affect the psychophysiological measures of subjects. In 2009, their results indicated that HR as a measure of arousal is a good correlator with self-report measures of player experience, both positive and negative [3], [10]. Going deeper into the topic, in 2010 they found that higher HR average is indicative of players feeling tense and frustrated. In general, a low HR average indicate positive affect, achieving the flow-state, feelings of competence and immersion and low levels of challenge [5].

\section{USER EXPERIENCE IN VIDEO-GAMES}

User experience assessment in electronic entertainment (EE) is currently done mostly by focus groups or by developer evaluation using subjective estimations. We feel that the development process might benefit largely from evaluating particular sections of the product (be it levels in videogames, or segments of movies) in a reliable way. As stated above, questionnaires in focus groups have several drawbacks regarding reliability, such as personal bias or untruthfulness of subjects. We propose a solution taking advantage of the autonomous nervous system (ANS), which functions automatically.

Due to different mindsets of players or video-games consumers, some classification is needed prior to the optimization of particular experiences.

\section{A. Single-player}

Single-player games provide a clear view on how various game events and situations affect the player's psychophysiological state (in comparison with multi-player games). Lacking the social aspect of the game makes players less distracted and more focused on the gameplay than playing with other people.

Single-player games provide a straight forward opportunity to use data for the evaluation of individual levels of the game. Unlike multi-player games, players encounter similar situations throughout the gameplay, which, for example may provide information on what sections of particular level are not entertaining enough.

\section{B. Multi-player}

The multi-player format provides a different environment for psychophysiological measurements. Players are set into the same game environment repeatedly, however the gameplay itself is rarely similar to the previous attempts. In addition, different player compositions create a dynamic setting which makes measurements considerably more difficult than in the single-player format.

The game genre has a major influence on determining the player's psychophysiological state.

Video-game genres can be classified according to several factors. Typical established genres are: Action, Adventure, Role-playing, Simulation, Strategy, Sports and others. However, taking social factor into consideration, the classification of game genres may become more complex. For example, Fig. 1 shows a conceptual map of game genres in relation to social interaction and complexity of the game. It should be noted 
that the conceptual map still lacks a few game genres, such as simulator games.

Social aspect of games also has a significant effect on the nature of our experiments. For example, while playing a single-player game, the player's experience is not influenced by behavior or the performance of his/her teammates or opponents. In contrast, many multi-player games encourage their players to communicate with their teammates to simulate reallife battlefield experience or to improve their cooperation skills during gameplay. After initial set of experiments (described in VI), we have decided to use only single-player games in the future.

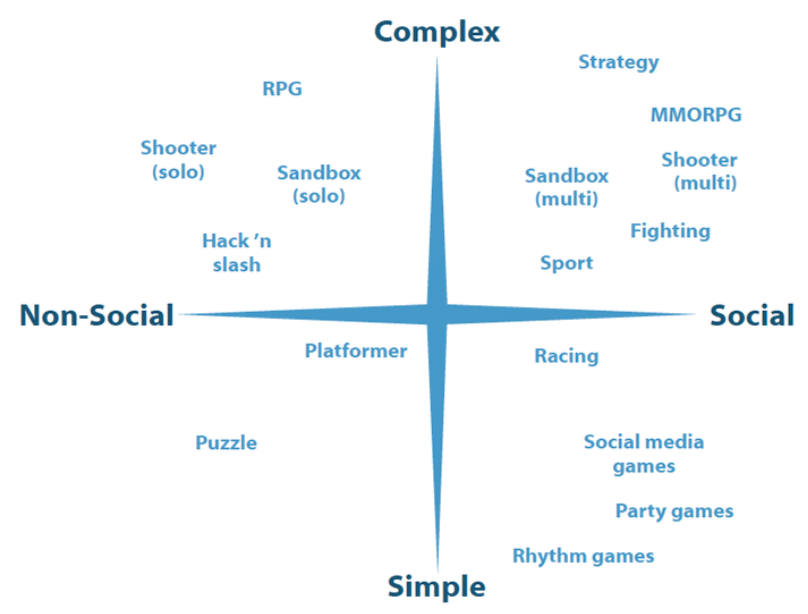

Fig. 1. Conceptual map of game genres in relation to social interaction and complexity of the game [11].

\section{PSYCHOPHYSIOLOGICAL MEASURES}

Any research method in which the dependent variable is a physiological measure and the independent variable is behavioral or mental (such as memory) is a psychophysiological method. Physiological measures take many forms and range from blood flow or neural activity in the brain to heart rate variability and eye movements. These measures can provide information about processes including emotion and cognition, as well as interactions between them. Physiological measures thus offer a very flexible set of tools for researchers to answer questions about behavior, cognition, and health [12].

All the measurements used in our research are parts of the human nervous system, more specifically the autonomic nervous system (ANS), which controls involuntary physiological responses. Measures controlled by the ANS function automatically. Therefore, they can not be affected or controlled consciously, which reduces the risk of unsuccessful experiments or imprecise results. We believe that a combination of traditional techniques with psychophysiological measurements will provide a reliable insight into player experience. Although there is no prior verification of reliability of such a combination, interviews and questionnaires are currently the only way to evaluate player experience, and therefore the only way to create our model. This section is a short introduction of how the ANS and psychophysiological measures used in our work function.

\section{A. Heart rate + Heart rate variability}

Heart rate (HR) is a measure of cardiovascular activity which reflects emotional state. It has been found to increase for a number of negative emotions (e.g. anger, anxiety, embarrassment, fear, sadness) as well as for some positive emotions (e.g. happiness, joy) and surprise [13].

The heart rate variability (HRV) is the time difference between each successive heartbeat, otherwise known as the $\mathrm{R}-\mathrm{R}$ interval or the inter-beat interval. The time between each heartbeat is not fixed or consistent, but it varies with every beat - hence the term variability.

Historically, HRV has been measured using an electrocardiogram (ECG), but with the development of technology, it can now be reliably measured using smartphone applications combined with a heart rate strap [14] or a pulse sensor [15]. Whilst there are many other metrics used to measure HRV, the most common is known as the "root-mean square difference of successive normal R-R intervals (RMSSD)" [16].

\section{B. Respiratory activity}

Respiration is measured as the rate of volume at which an individual exchanges air in their lungs. Previous research has found that respiration rate is increased by emotional arousal and decreased by rest and relaxation [17]. Overall, respiratory activity (RA) is rather easily measured during the experiments. There are several types of devices used for measuring respiratory activity, worn on the chest, torso, neck or even the wrist.

\section{Electrodermal activity}

Electrodermal activity (EDA) measures the activity of the eccrine sweat glands and has been found to be a linear correlate to arousal [18]. Although room temperature, humidity, participants activities and the correct attachment of the electrodes have to be carefully considered, tonic EDA is a well researched and valid method to record arousal and has been used for measuring emotions for interaction with systems [19], [20]. EDA sensors are typically worn at the fingertips, but nowadays wrist-worn sensors and even ring sensors are becoming available.

\section{Methods}

In order to determine an overall experience of the subject we need to be able to model relations between changes in psychophysiological measures and the subject's emotional states. Initial experiments (Sec. VI) have already proven that using absolute values of psychophysiological measures is not practical due to major variability in readings connected to the subject's states prior to the experiment. Instead, the deviation from average values of the subject in each experiment session will be used.

Furthermore, a possibility of previous states affecting the subject's present emotional state procure the need to take 
these into account. Methods such as Hidden Markov Models, Neural Networks with Long Short-term Memory architecture or Random Forests pose as suitable candidates for our research. Since our research will recline upon analyzing timeseries data, using Hidden Markov Models should prove useful for their ability to discover temporal patterns in sequential data. With physiological changes taking place over time, we suppose that investigating our data in sequences may help us find correlations between psychophysiological states of subjects and their gameplay experience. Similarly, we plan to use LSTM Neural Networks for their ability to take longterm dependencies into account. Additionally, Random Forest or Decision Trees may provide insight into relations between individual changes in measures. If such relations are shown, these methods may produce a rule-based knowledge showing correlations between individual measures.

\section{A. Subject sample}

A lack of resources would probably pose a problem should we want to use a representative sample of the whole population in our research. Hence the subject sample in our research will be adjusted to serve the needs of video-game developers in order to cover as much of present video-games target demographic as possible. Elliott et al. explained variations in demographic characteristics by video game genre [21]. In 2012, the European summary report presented profile of average gamer, as well as statistical overview of age and gender incidence of gaming [22]. We plan to consider the results of their research in the process of creating our personalized questionnaire.

\section{B. Hidden Markov Model}

Hidden Markov Model (HMM) is a powerful statistical tool for modeling generative sequences that can be characterized by an underlying process generating an observable sequence. HMMs have been successfully applied in many areas related to signal processing, and in particular speech processing, as well as low level natural language processing tasks such as part-of-speech tagging, phrase chunking, and extracting target information from documents [23].

Even though HMMs are mostly used for time-series data prediction, we expect them to perform well as an analysis tool in our research. Török et al. used HMM in similar research into time-series data analysis with a focus on emotional states of subjects watching a short animated movie [24].

\section{Random forest}

In 2001, Breiman proposed random decision forests, which added an additional layer of randomness to bagging of decision trees. In addition to constructing each tree using a different bootstrap sample of the data, random forests change how the classification or regression trees are constructed. In standard trees, each node is split using the best split among all variables. In a random forest, each node is split using the best among a subset of predictors randomly chosen at that node. This somewhat counterintuitive strategy turns out to perform very well compared to many other classifiers, including discriminant analysis, support vector machines and neural networks. In addition, random forests proved themselves robust against overfitting [25].

\section{Long Short-Term Memory Neural Network}

Unlike traditional RNNs, an LSTM neural network is wellsuited to learn from experience to classify, process and predict time series when there are very long time lags of unknown size between important events. The LSTM contains special units called memory blocks in the recurrent hidden layer. The memory blocks contain memory cells with self-connections storing the temporal state of the network in addition to special multiplicative units called gates which control the flow of information. Each memory block in the original architecture contains an input gate and an output gate [26]. Later, the forget gate was added to the memory block [27].

\section{E. Self-reporting}

In addition to measuring psychophysiological responses of subjects, we also need to use self-reporting methods to create a training data set for learning the user experience model. Questionnaires and interviews have proven to be useful selfreporting measures and are used world-wide for assessing userexperience in different fields. Previously, researches have studied whether self-reporting correlates with psychophysiological measurements and whether the subject's answers are valid even if disagreeing with measured values [28], [29]. We plan to use a simplified version of iGEQ questionnaire, mentioned in II.

\section{INITIAL EXPERIMENTS}

This section describes our initial set of experiments along with our observations and a few notes on the experiments that may be useful for future research. These experiments were not done in order to create a ready-to-use user experience model itself; rather, their purpose was to provide an initial insight and hypotheses for future research. However, a preliminary analysis of our data shows the existence of a correlation between psychophysiological measurements and an in-game event.

Using psychophysiology, it is crucial to consider carefully prior psychophysiological states of the subject. As described in [9] as well as found by our initial experiments, particular readings of subjects vary, depending on several factors. For example, in Fig. 2 there are 4 games of a subject player in separate sessions during three days. The figure shows that the average HR of the subject may vary significantly, due to factors affecting the subject prior to the experiment, such as caffeine intake, emotional state, sleep quality, hormonal changes, etc. Besides the psychophysiological state, the game genre and format also affect experimental readings considerably. While in Single-player games players are focused solely on game experience, in Multi-player format the social aspect comes into play. And while our initial findings show that HR is not affected by verbal communication with teammates and 
opponents (Sec. VI-B), this does not have to apply to other measures that will be used in experiments. Therefore we decided to ignore absolute values of HR, RA and EDA measurements in the future and use only deviations from the average values.

\section{A. Dota 2}

After 18 games of Dota 2, 9 out of which won, the subject showed an average $\sim 83 \mathrm{HR}$. The subject averaged $\sim 84 \mathrm{HR}$ during the winning games and $\sim 81$ average HR while losing. With a larger sample size, we will be able to determine whether losing or winning a game has an impact on the subject's HR.

As described in Sec. III-B, it has to be taken into consideration that recreating the same conditions for multi-player experiments is a near-to-impossible task, especially when experiments are done with longer games such as those in Dota 2 , since it is difficult to play several games in a row on the same day.

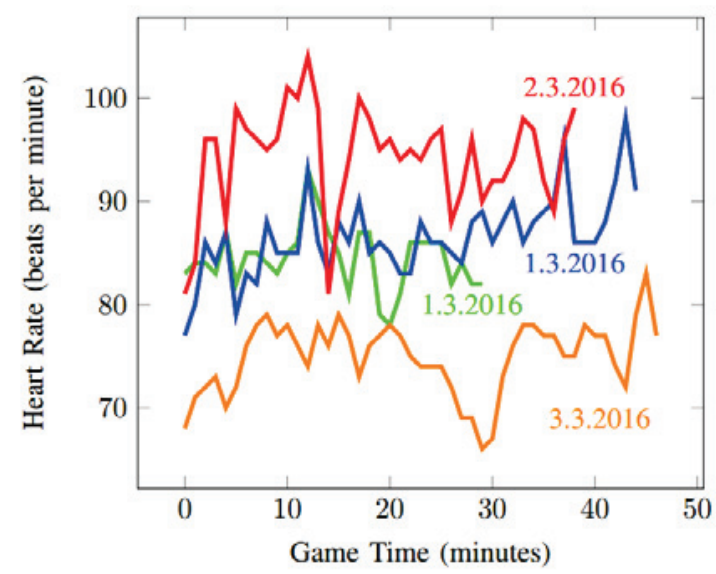

Fig. 2. 4 games of Dota 2 played by the tested subject in 3 sessions.

Fig. 2 displays similarities of the subject's HR during several games of Dota 2, even though games were played in several different sessions. There are noticeable spikes of HR in several game stages.

After only a small number of games played, we were already able to find some interesting patterns emerging from our experiments. There are several spikes in the typical game of Dota 2, where the player is participating in massfights (i.e. in-game clashes of multiple players from both teams). It is obvious that the HR of the player is rapidly decreasing while the player is dead (waiting for respawn).

Fig. 3 shows HR of the subject during the game of Dota 2.

In every game, there is also a significant spike in HR of player at the end of the game (see Fig. 3).

\section{B. Hearthstone}

The subject played 3 sessions of Hearthstone games, one consisting of 4 games, the other two of 2 games. The subject's

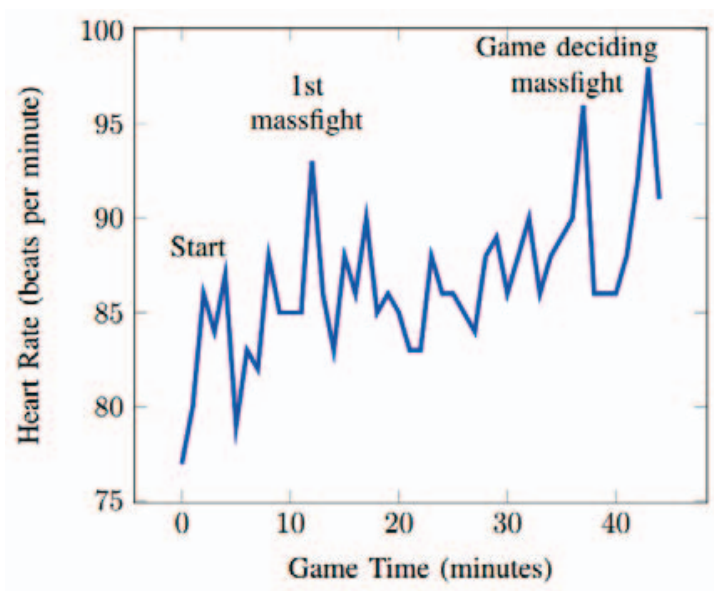

Fig. 3. Typical game of Dota 2 played by the tested subject.

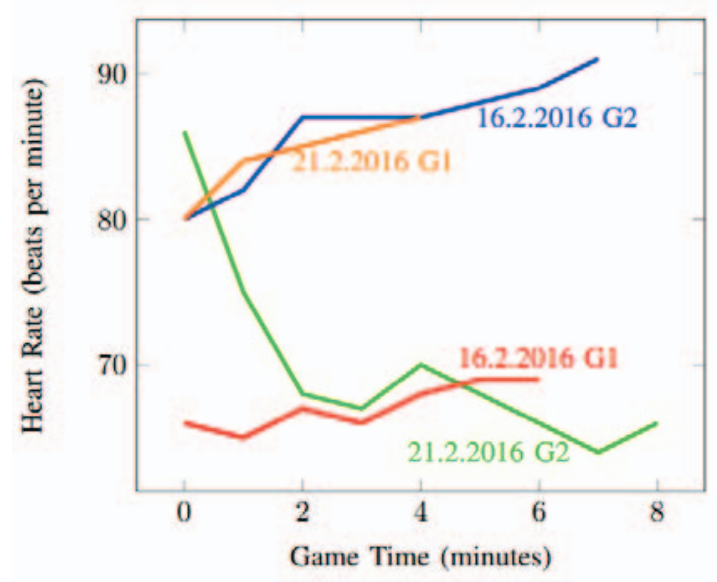

Fig. 4. Hearthstone sessions 1+2.

average HR during the first two sessions was 76.97 (see Fig. 4).

The third session showed average HR of 82,69 (see Fig. 5).

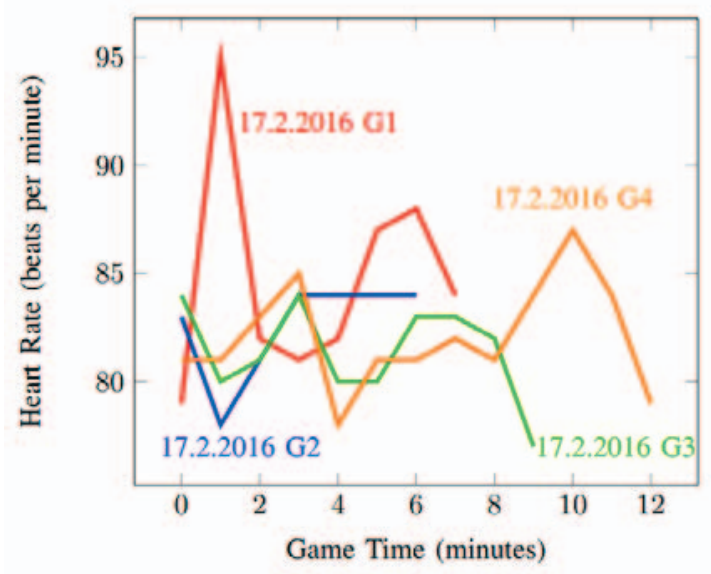

Fig. 5. Hearthstone session 3. 
Both Fig. 4 and Fig. 5 show the subject's HR during different Hearthstone games, played in different sessions. There are no easily observable patterns due to the small number of experiments, yet this is to be changed in further research.

Of course, while one Dota 2 game lasts roughly about 40 minutes to 1 hour on average, the typical Hearthstone game lasts for around 5-10 minutes. This has to be taken into account, since it is unlikely to preserve above-average (or below-average) HR for a long time during the game. There are no obvious patterns visible from such a small number of experiments (compared to Dota 2), which is most likely caused by the short and non-dynamic nature of Hearthstone games. The results seem chaotic at first, but we are confident that after obtaining a larger dataset, some interesting patterns will be uncovered.

After analyzing the patterns we found in our initial experiments we can divide them into two categories:

1) Game-specific patterns: As stated above in this section, there are regular spikes in the subject's HR during the game of Dota 2.

2) Psychophysiological patterns: Even though there are several factors affecting psychophysiological state of the subject, we have found interesting readings dependent on the subject's current physical state. For example our experiments have shown fairly different HR values depending on the time of the day. During the night (00:00 AM - 04:00 AM), the subject averaged up to 20 less HR than during the day. Surprisingly, the subject did not show much variation in HR after being emotionally involved during the game (e.g. verbal confrontation with teammates/opponents).

\section{FUTURE WORK}

After initial phase of our work and experiments, we created a system for collecting physiological data from three different sensors. We collect data from three different sensors to gather 10 physiological measures:

- Bioharness 3 is a chest strap sensor which collects 5 different physiological measures, such as Heart Rate + Amplitude, Respiratory Rate + Amplitude and Activity.

- Mindwave Mobile is a head-worn sensor gathering simplified EEG data. Furthermore, our software uses raw EEG data to output calculated values of Attention and Meditation which we use as features in our research. Additionally, we use raw data to calculate average number of eye blinks in past minute.

- GSR sensor is a fingertip sensor for collecting GSR conductance and resistance data.

Upon collecting the data, we conduct an interview with every subject to label different sequences in game on scale from 1 to 5 based on their overall enjoyment. This evaluation is stored in fun column and poses as key feature in our machine learning model. Sample data are shown in Fig. 6.
To this date, we have conducted approximately 20 hours of experiments with 15 participants. We plan to add $\sim 15$ another subjects before training our final user experience model.

\section{Conclusion \& Future Challenges}

This paper proposes a potentially promising direction for future research and presents an introductory analysis of available physiological data sources, their relevance to user experience modeling and technical prerequisites for their collection. During our initial experiments, one subject participated in 18 games of Dota 2, consisting of approx. 20 hours of gameplay. We discovered a correlation between in-game events and physiological changes of the subject, which suggests a possibility to model the user experience in electronic entertainment based on physiological measurements. These experiments, although using only HR measurements, exposed several interesting patterns concerning both game-specific events as well as overall useful facts about the measurements. We repeatedly detected increased HR in specific game situations (e.g. massfights, first contact with opponent) or decreased HR during the times the subject waited for his character to respawn after death.

In future research, we plan to increase the number and extent of the experiments substantially and use the findings for creating a model of user experience in correlation with the psychophysiological state of the EE consumer. However, as stated in Sec. I, simply measuring psychophysiological states of the subject is not sufficient for a successful training of user experience model. We plan to combine these measurements with specifically designed interviews as well as video recordings of the subject's gameplay in order to determine the subject's experience. As stated in IV, to create the model, we have to rely initially on the above-mentioned questionnaires and interviews. With this information, experiments will pose as a training set for our model, with the goal to predict the experience of players in the future.

\section{A. Future Challenges}

The initial set of experiments have exposed several challenges related to our future research plans:

Finding a suitable subject sample for our experiments. As previously stated, we plan to cover most of the videogames market demographic, in order to fulfill our goal of optimizing the consumer's experience in electronic entertainment.

Correlation vs causation: We realize that even after finding correlations in multiple psychophysiological data and the player's psychophysiological state, it will not provide evidence of their experience affecting these measures. However, with a sufficient amount of experimental data, we are confident about creating a working user experience model to be used in the development process. 

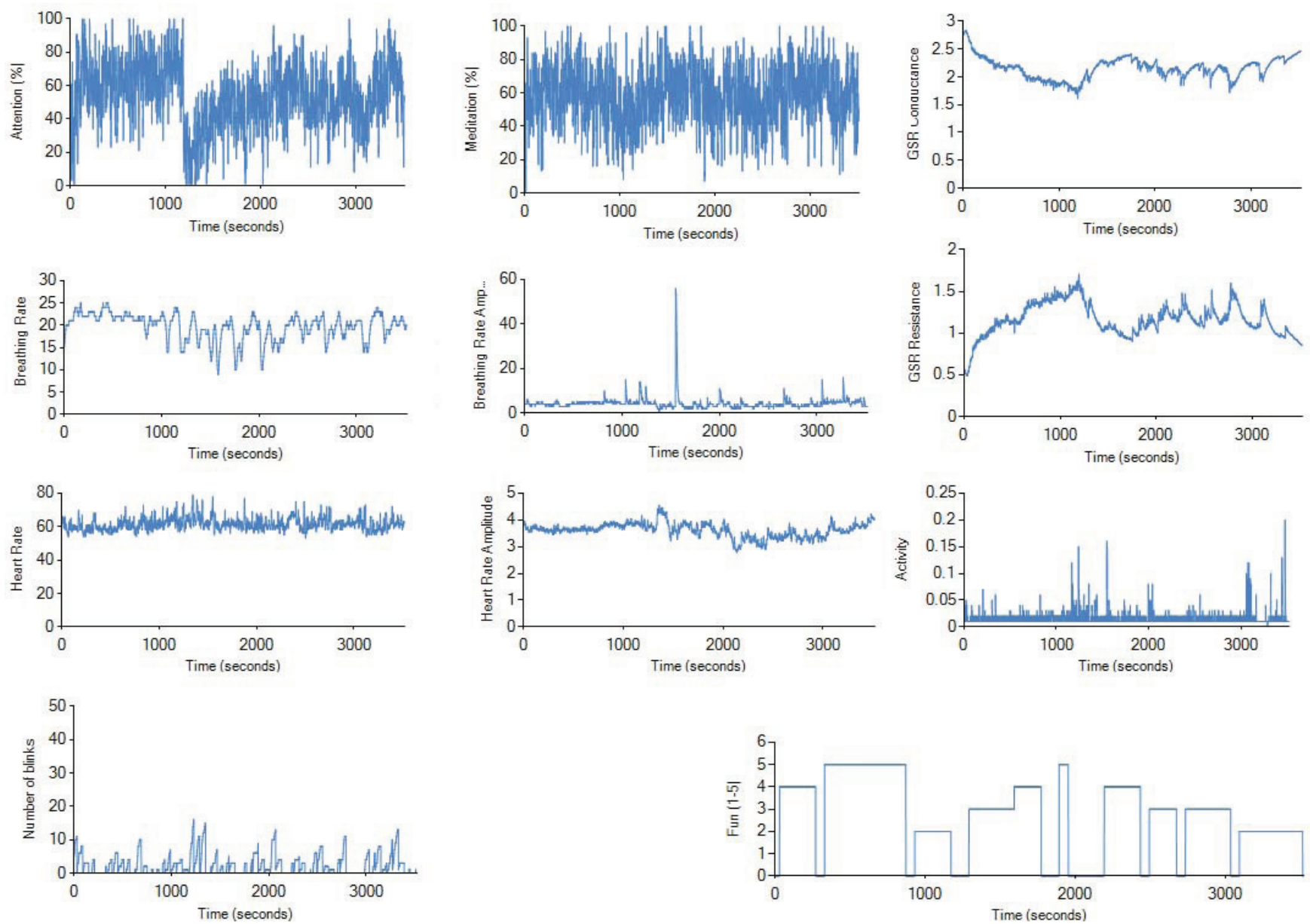

Fig. 6. Sample data from data collection software

During the experiments, concerns arose regarding the accuracy of the selected sensors for experiments. With non-medical grade sensors, such as we propose to use, there is a risk of inaccurate readings during gameplay. However, the experiments have shown that accuracy of commercially available sensors can be sufficient, especially if only deviations from average readings will be used in our research.

As described in section VII, we plan to conduct additional $\sim 20$ hours of experiments where we collect 10 physiological measures prior to training our final user experience model.

\section{REFERENCES}

[1] E. D.E.S.A. Washington, "Sales, demographics and usage data Essential facts about the computer and video-game industry," 2015.

[2] J. M. Kivikangas, G. Chanel, B. Cowley, I. Ekman, M. Salminen, S. Järvelä, and N. Ravaja, "A review of the use of psychophysiological methods in game research," Journal of Gaming \& Virtual Worlds, vol. 3, no. 3, pp. 181-199, 2011.

[3] A. Drachen, L. E. Nacke, G. Yannakakis, and A. L. Pedersen, "Psychophysiological correlations with gameplay experience dimensions," arXiv preprint arXiv:1004.0243, 2010.
[4] W. IJsselsteijn, W. Van Den Hoogen, C. Klimmt, Y. De Kort, C. Lindley, K. Mathiak, K. Poels, N. Ravaja, M. Turpeinen, and P. Vorderer, "Measuring the experience of digital game enjoyment," in Proceedings of Measuring Behavior. Noldus Information Tecnology Wageningen, Netherlands, 2008, pp. 8889.

[5] A. Drachen, L. E. Nacke, G. Yannakakis, and A. L. Pedersen, "Correlation between heart rate, electrodermal activity and player experience in first-person shooter games," in Proceedings of the 5th ACM SIGGRAPH Symposium on Video Games. ACM, 2010, pp. $49-54$.

[6] M. E. Ballard and J. R. Wiest, "Mortal kombat (tm): The effects of violent videogame play on males' hostility and cardiovascular responding1," Journal of Applied Social Psychology, vol. 26, no. 8, pp. 717-730, 1996.

[7] M. Codispoti, P. Surcinelli, and B. Baldaro, "Watching emotional movies: Affective reactions and gender differences," International Journal of Psychophysiology, vol. 69, no. 2, pp. 90-95, 2008.

[8] K. P. Kallio, F. Mayra, and K. Kaipainen, "At least nine ways to play: Approaching gamer mentalities," Games and Culture, p. 1555412010391089, 2010.

[9] R. L. Mandryk, K. M. Inkpen, and T. W. Calvert, "Using psychophysiological techniques to measure user experience with entertainment technologies," Behaviour \& information technology, vol. 25, no. 2, pp. 141-158, 2006.

[10] G. N. Yannakakis, J. Hallam, and H. H. Lund, "Entertainment capture through heart rate activity in physical interactive playgrounds," User Modeling and User-Adapted Interaction, vol. 18, 
no. 1-2, pp. 207-243, 2008.

[11] I. Granic, A. Lobel, and R. C. Engels, "The benefits of playing video games." American Psychologist, vol. 69, no. 1, p. 66, 2014.

[12] Z. Infantolino and G. A. Miller, "Psychophysiological methods in neuroscience," http://nobaproject.com/modules/ psychophysiological-methods-in-neuroscience, accessed: 201603-15.

[13] S. D. Kreibig, "Autonomic nervous system activity in emotion: A review," Biological psychology, vol. 84, no. 3, pp. 394-421, 2010.

[14] A. A. Flatt and M. R. Esco, "Validity of the ithletetm smart phone application for determining ultra-short-term heart rate variability," Journal of human kinetics, vol. 39, no. 1, pp. 85-92, 2013.

[15] J. A. Heathers, "Smartphone-enabled pulse rate variability: an alternative methodology for the collection of heart rate variability in psychophysiological research," International Journal of Psychophysiology, vol. 89, no. 3, pp. 297-304, 2013.

[16] A. E. Aubert, B. Seps, and F. Beckers, "Heart rate variability in athletes," Sports medicine, vol. 33, no. 12, pp. 889-919, 2003.

[17] R. M. Stern, W. J. Ray, and K. S. Quigley, Psychophysiological recording. Oxford University Press, USA, 2001.

[18] M. E. D. A. M. Schell and D. L. Filion, "The electrodermal system," Principles of Psychophysiology: Physical, social and inferential elements, pp. 295-324.

[19] J. McCarthy and P. Wright, "Technology as experience," interactions, vol. 11, no. 5, pp. 42-43, 2004.

[20] R. D. Ward and P. H. Marsden, "Physiological responses to different web page designs," International Journal of HumanComputer Studies, vol. 59, no. 1, pp. 199-212, 2003.

[21] L. Elliott, A. Golub, G. Ream, and E. Dunlap, "Video game genre as a predictor of problem use," Cyberpsychology, Behavior, and Social Networking, vol. 15, no. 3, pp. 155-161, 2012.

[22] I. MediaCT, "Videogames in europe: Consumer study. european summary report," 2012.

[23] P. Blunsom, "Hidden markov models," Lecture notes, August, vol. 15 , pp. 18-19, 2004

[24] A. Török, "Time series analysis in r: using hidden markov models for unsupervised learning [satrday, 3 september 2016]," https://www.youtube.com/watch?v=3N18Y2y2jgk\&index $=15 \&$ list=PLUB10DoLa5SDsOetNga5pt4uo6YOyKVWn, accessed: 2017-01-23.

[25] L. Breiman, "Random forests," Machine learning, vol. 45, no. 1, pp. 5-32, 2001.

[26] H. Sak, A. W. Senior, and F. Beaufays, "Long short-term memory recurrent neural network architectures for large scale acoustic modeling." in Interspeech, 2014, pp. 338-342.

[27] F. A. Gers, J. Schmidhuber, and F. Cummins, "Learning to forget: Continual prediction with lstm," Neural computation, vol. 12, no. 10 , pp. $2451-2471,2000$.

[28] G. H. Gudjonsson, "Self-reported emotional disturbance and its relation to electrodermal reactivity, defensiveness and trait anxiety," Personality and Individual Differences, vol. 2, no. 1, pp 47-52, 1981

[29] A. De Los Reyes, A. Aldao, S. A. Thomas, S. Daruwala, A. J. Swan, M. Van Wie, K. Goepel, and W. V. Lechner, "Adolescent self-reports of social anxiety: Can they disagree with objective psychophysiological measures and still be valid?" Journal of Psychopathology and Behavioral Assessment, vol. 34, no. 3, pp. 308-322, 2012. 